\title{
The Drawn Space for Inclusion and Communicating Space
}

\author{
Anna Lisa Pecora
}

\section{Abstract}

In the last twenty-five years, some studies have been analyzing the virtual reality potential for special education. They show the virtual environments as a valid communicative medium and a safe space where ASD people can experience new situations without limits of 'in vivo' experiences. Often, problems with space can complicate many aspects of everyday life. Referring to Hermelin and O'Connor's studies, the difficulties involving the autistic clinical frame are connected especially to perception deficiency, therefore the VR can become valid support, for people with ASD, improving relationships with space, with ourselves and with others. My study tries to provide a guideline tool for a humancentered $\mathrm{VR}$ design.

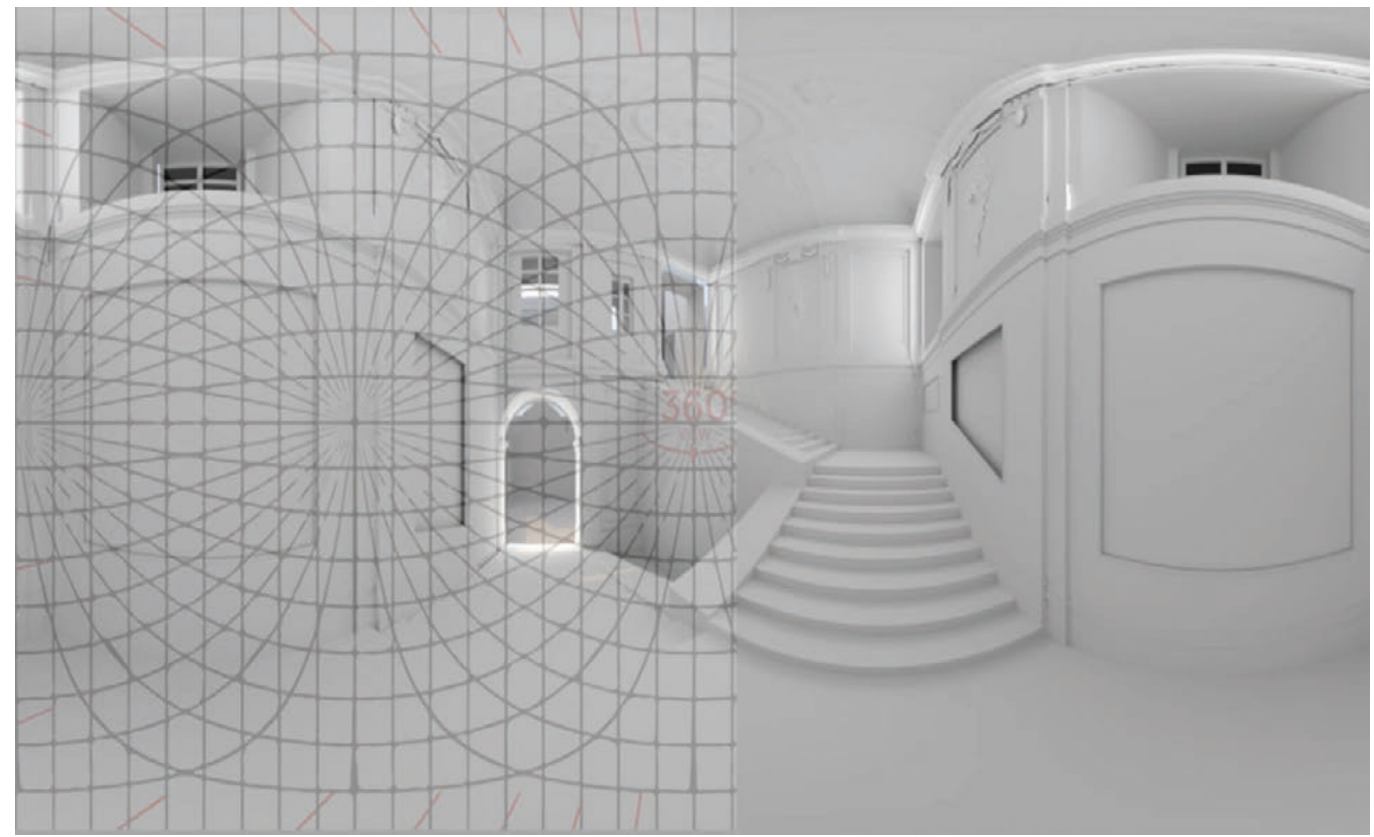




\section{Introduction}

In recent years, the drawing disciplines, supported by the widespread of new technologies progress, play a fundamental role in the interdisciplinary mediation of the communicative field. Up to now, studies about special education and VR have been led by medical and psychological disciplines, disregarding perceptual aspects about the relationship between the user and the virtual space. Furthermore, a wrong interpretation of spatial configuration can provide various types of discomfort in both, typical and non-neurotypical users. If $\mathrm{VR}$ can mediate the relation with the real world, its visual codes must be managed with expertise, in order to provide a customized visual language on specific user needs. Therefore, the ICAR 17 disciplines can embody a valid guide to drive the perceptual process in virtual reality.

\section{VR Spaces for Learning and Inclusion}

In the last twenty-five years, the rapid development of virtual reality technology has improved the simulation quality, opening new applicative frontiers. Their communicative power lies in the potential to provide a perceptual realistic experience where it's possible to activate the same cognitive dynamics available in the real world. Therefore, in special education, the immersive experience should support the specific characteristics of the autistic perceptual system and its atypical responses to sensorial stimuli. Some impairments can create visual distortion complicating the reading of spatial clues; for example, they can inhibit the understanding of depth, dimensions, shapes and relative positions [Bogdashina 2015, p. 29]. Other problems can occur in recognizing the space limits or in the relation between the viewer's body and other objects. This problem can arise during a VR experience due to the specific characteristics of virtual navigation. Virtual reality uses panoramic images, also called 360 degrees images, as: photorealistic renders, photos or videos; therefore, since the frame limits exceed the visual field limits, the experience involves not only the vision but also the body, even in the simple head rotation. This way, the user turns from a static sight to a dynamic perspective [Rossi et al. 2019]. Usually, these relations between vestibular and visual apparatus help the wayfinding and the understanding of the space, but in autism, they can provide confusion and stress due to the disorder of the "afferent couple" phase [Russell 1994]. For this reason, the majority of the research about VR and autism, attempt to create a safe space where experience new situations without the limits of the physical world. Cultural heritage, for example, museums or archeological sites, often are uncomfortable spaces for autistic people, because they can provide overstimulation of senses, causing a painful and stressful experience for people with hypersensitivity. Using the potential of virtual reality, it's possible to communicate cultural information customizing the input data on the specific user needs helping, this way, the dialogue between the space and the viewer. Moreover, since the virtual experience involves the user in the first person like in the real world, the emotional aspects play a strategic role, enhancing the learning process with a playful incentive. In fact, most of the learning experience for special education use applications from the gaming world, without customizing perceptual inputs on autism needs. Therefore, the research lacks in details concerning useful aspects about the perceptive response to the stimulus coming from the designed virtual space. Most of the observed studies are visually overloaded and unfitted to the cognitive needs of ASD people. The majority of research, in fact, points out psychological and technical aspects without considering visual factors involved in the interaction between autistic people and VR. Trough the comparison between the scientific researches published about VR and autism, I identified four taxonomic categories in order to analyze the state of the art about this topic:

Usability tests: valuating ASD users' answers.

Life skills: learning of basic competencies useful in daily life.

Social skills: understanding of social situations.

Special education:VR application in schools environment, mainly for language learning. 
Fig. I. Cobb's experiment (1995). Frame of the virtual ski slope from the user's point of view and scheme with the main depth clues and target elements. Graphic drafting by Anna Lisa Pecora.

\section{Visual Clues Improving Space Perception}

There is a huge difference between watching a real space and watching a drawn space. In the first case, space directly communicates its morphology without interferences, while, watching an image means to see a subjective interpretation of reality. Also, the virtual environment develops by a drawing action, therefore the perceived image is double filtered: first by the designer, who translates his personal view of the world in graphical signs, then by our brain, that, according to the personal experience, decode the figurative codes in a mental image. Thus, the final result depends on multiple factors, some depending on the person, others on the picture characteristics. Usually, we distinguish three main categories collaborating to the space interpretation and, in detail, to the depth: pictorial depth cues [I], physiological depth cues, motion depth cues. Jerald Jason adds another category: contextual distance factors, related to the environment and psychological influence on our perceptual behavior [Jason 2016, pp. 122-123]. He explains that: "perception and action are closely intertwined. Action-intended distance cues are psychological factors of future actions that influence distance perception" [Jason 2016, p. 122]. This aspect is particularly relevant if re-
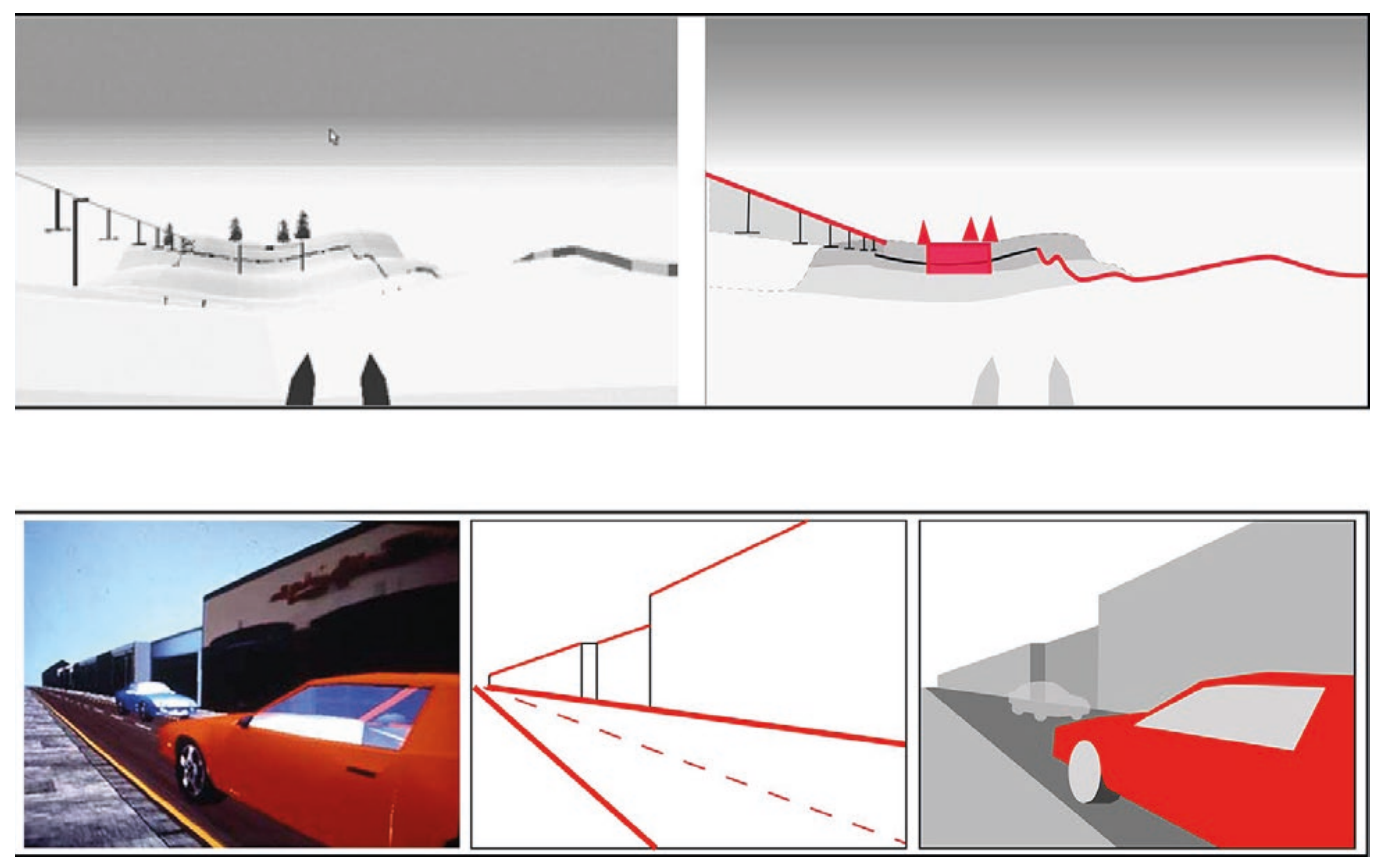

ferred to autism where the answer to sensorial stimuli can be altered by an atypical behavior of the perceptual system. Moreover, this impairment can cause states of anxiety and fear, interfering in the right reading of depth cues. Consequently, designing a virtual space, it's important to take into account psychological aspects influencing physiological and projective factors when managing graphical signs. For example, the environment should be characterized by simplicity and clear spatial clues. Referring to this aspect, an example is one of the first experiments led by Sue Cobb and the VIRART team. There are no descriptions of the virtual environment, but, watching the published photos, it is possible to deduce some visual aspects interfering in the perceptual process. The VE represents a ski slope characterized by a simple framework where objects have basic geometries easily recognizable (fig. I). The highly prevalent white produces a flat configuration where it's difficult to understand the perspective. For this reason, the use of some graphic depth cues is crucial: a curb on the right and a schematic ski lift on the left side, provide two linear clues that, converging toward the frame center, become important depth gradients. In fact, the angle of graphic signs on the vertical and horizontal axis is a key element for tridimensional perception [Arnheim 1997]. The distances and dimension reduction of ski-lift elements offer other depth gradi- 
ents in the observed image; acting in the same direction, they enhance each other because "the more the gradient is regular, the stronger their effect acts" [Arnheim 1997, p. 227]. We can find another example of communicating virtual space in Strickland's experiment, published in 1997; that is one of the first immersive experience for autism lead with an RV-HMD. It could be taken as a reference for its way to put in evidence target elements and depth clues. Strickland, describing the environment design, explains that: "The virtual world was a simplified street scene consisting of a sidewalk and textured building shapes. All motion objects such as people, animals, and objects in the sky were removed. Periodically one car, whose speed could be changed, would pass the child standing on a sidewalk. The contrast was kept low in the scenes with gray being the dominant color. The low quality of the headset screens provided a less detailed environment automatically. The cars, the focal point of the test, were presented in bright, contrasting colors [...] red and blue" [Strickland Dorothy 1997, p .4]. Only later, another visual stimulus is introduced: a stop sign is moved to different parts of the tracking area during the later tests and the children are asked to find it and stopping there (fig. 2). In this kind of configuration, the environment works like a neutral background helping to focus on visual targets. Otherwise, the oversimplification of morphological spatial signs creates difficulties in evaluating distances. It's possible to improve the information about the spatial reference using some graphic solutions without exceeding in perceptual overload; for example, acting on the textures or using some objects like landmarks in the scene. Sue Cobb's experiment, in 2002, uses a grid pattern for the virtual cafè floor, that works like a spatial coordinates reference. Moreover, they found that watching part of the avatar's body, enhances the sense of embodiment and supports the understanding of the avatar's size during motion [Cobb 2002, p. 17]. The sense of embodiment, is related to "presence", also defined as "the sense of being there", "inside a space, even when physically located in a different location" "[lason 20 I 6, p. 46]. Because ASD people frequently show proprioception impairments, they have difficulty feeling their body physically acting in the virtual environment as if they were performing the task in the real world. Giving the human height to the point of view, provides a familiar appearance to the framework improving the sense of presence and the willingness toward the virtual experience. For the sake of narration, I could only describe here a brief example of the guideline tool I compiled for designing an autism-friendlyVR. The attached tables (fig. 3) show part of the developed tool.
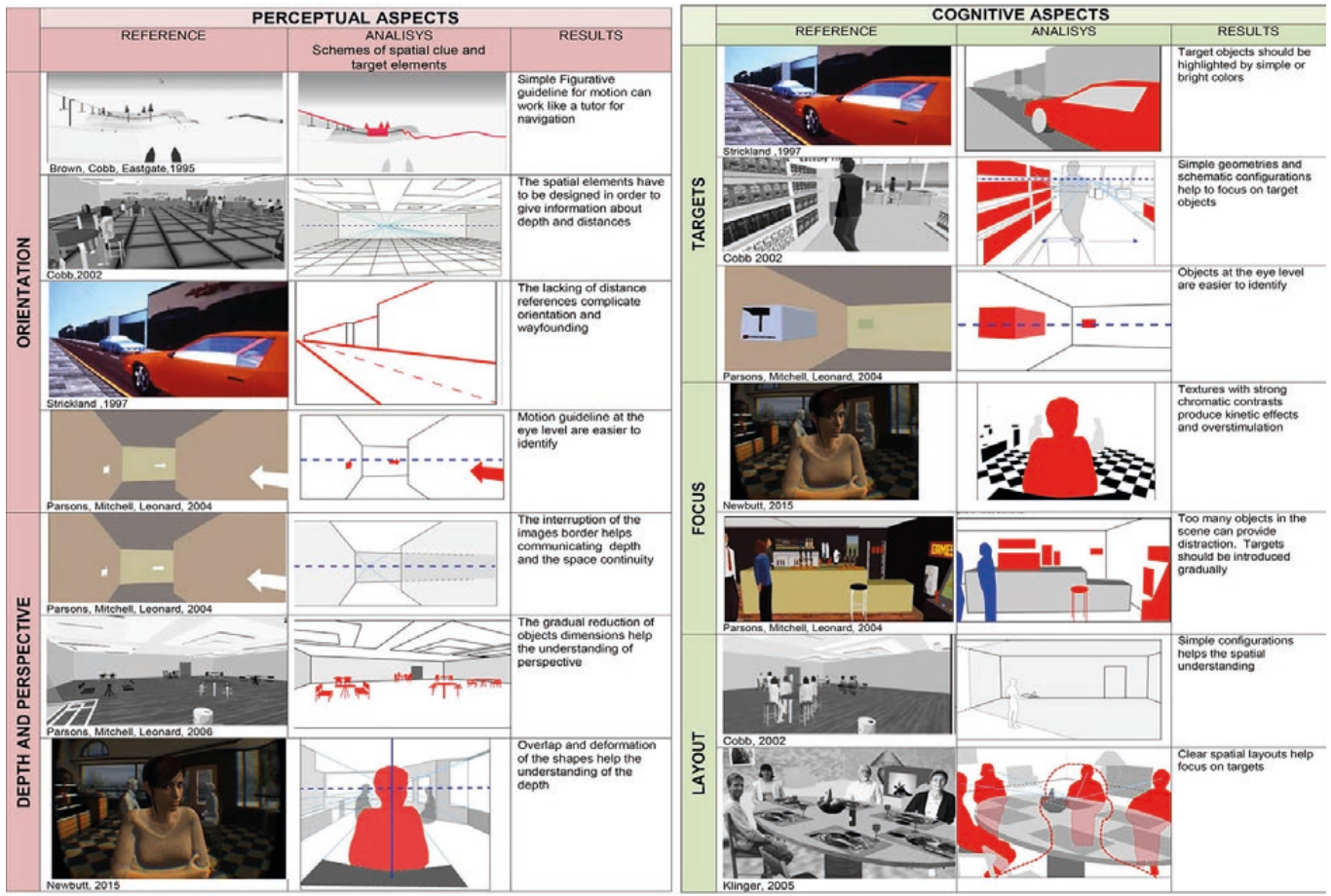


\section{Conclusions}

The physical space holds the complex theoretical and morphological relations between different elements as colors, lights, textures and patterns. So, only through the right graphic choices, their figurative configuration can be deeply understood. Setting the detail level, the chromatic and luminous qualities, the quantity and the value of graphic signs in the VE, become essential features to define the sense of immersion and presence and, therefore, the communicative power driven by perceptual inputs.

As Olga Bogdashina asserts, "filtering of an infinite amount of information is necessary to make the processing of information effective and conscious" [Olga 20 I5, p. I I0]. For this reason, the graphic design for an "autism-friendly" VR has to provide essential information about the environment to reduce sensorial weight avoiding distraction. In fact, the representation path allows the process of "synthesis, communication and explicitation" of the space, necessary for decoding and subsequent learning its cultural contents. The relationship with environment can influence human abilities to the point of providing knowledge for non-neurotypical people as well as for anybody else. A right interpretation of spatial codes through drawing disciplines can provide a human-centered design of a virtual environment where architectural space becomes the medium for communication and inclusion. In future studies, the guidelines introduced in the present paper will be used to produce a prototype to test them with the user reference.

\section{Notes}

[I] So called referring to human optical system, elaborating images by projection on the retinal surface.

\section{References}

Arnheim Rudolf (1997). Arte e percezione visiva. Milano: Feltrinelli. Trad. of Gillo Dorfles. Orig. ed. (1954) Art and Visual Perception: A Psychology of the Creative Eye. By the Regens of the University of California.

Bogdashina Olga (20I5). Le percezioni sensoriali nell'autismo e nella sindrome di Asperger.Vignate (MI): Uovonero, Trad. of Luca Brivio. Orig. ed. (2003), Sensory Perceptual Issues in Autism and Asperger Syndrome. Different sensory Experiences - Different Perceptual World. London: Jessica Kingsley Publishers Ltd.

Cobb Sue Valerie Gray, Beardon Luke, Eastgate Richard, Kerr Steven J. (2002). Applied Virtual Environments to support learning of Social Interaction Skills in users with Asperger's Syndrome. In Digital Creativity, I (I 3), pp. I I-22.

Jason Jerald (2016). The RV Book: Human centered design for Virtual Reality. New York: ACM Books.

Katz David (1992). La psicologia della forma. Torino: Bollati Boringhieri.

Marcolli Attilio (197|). Teoria del Campo I. Firenze: Sansoni.

Robertson Caroline E., Kravitz Dwight J., Freyberg Jan, Cohen-Simon Baron, Baker Chris (20 I3).TunnelVision: Sharper Gradient of Spatial Attention in Autism. In The Journal of Neuroscience, 17, pp. 6776-6781.

Rossi Daniele, Olivieri Alessandro (2019). First Person Shot: la prospettiva dinamica interattiva negli ambienti virtuali immersivi. In Riflessioni. L'arte del Disegno / II Disegno dell'Arte. Convegno UID 20 19. Roma: Gangemi editore International, pp. 977-984.

Russell James (1994). Agency and early mental development. In Bermudez José Luis, Eilan Naomi, Marcel Anthony (eds.). The body and the self. Massachusetts, London:The MIT Press Cambridge.

Sheppard Elizabeth, Ropar Danielle, Mitchell Peter (2009). Perceiving the impossible: How individuals with autism copy paradoxical figures. In Autism, 13 (4), pp.435-452.

Simmons David R., Robertson Ashley E., McKay Lawrie S., Toal Erin, McAleer Phil, Pollick Frank E. (2009). Vision in autism spectrum disorders. In Vision Research, 49, pp 2705-2739.

Strickland Dorothy (1997). Virtual Reality for the Treatment of Autism, Virtual Reality in Neuro-Psycho-Physiology. Amsterdam: los Press.

Author

Anna Lisa Pecora, Dept. of Architecture, University of Naples Federico II, annalisapecora@hotmail.com 
